\title{
Content-based image retrieval system for marine invertebrates
}

\begin{abstract}
There are many marine life around the world where it is essential to have proper documentation for future records. Many information retrieval systems for marine science today require text input from user and can only be accessed online. Therefore, people who do not know the name of the marine species or do not have Internet access cannot search using the systems. Responding to this important need, this work aims to develop a Content-based Image Retrieval (CBIR) system for marine invertebrates based on colour and shape features. With the CBIR system for marine invertebrates, users can use the system to look for marine invertebrates' species instead of using traditional methods of searching such as using books and encyclopedias. Users can easily upload the image of marine invertebrate that they want to search into the system and the system will retrieve all the other similar images of marine invertebrates along with their description. All the system interface's buttons, icons and text were designed in a way where any user can easily understand and further learn to operate the system themselves. Based on the retrieval effectiveness experiment and questionnaire-based survey, the proposed CBIR system for marine invertebrates is shown to be effective, help users search similar images of marine invertebrates, provide concise information on marine invertebrate's species for learning purposes, and is reliable and user-friendly.
\end{abstract}

Keyword: Content-based Image Retrieval; Marine invertebrates; Colour feature; Shape feature 